(PWID; range: 9.5-70.0\%; 95\% CI: 24.1-40.1\%), and 2.5\% among populations at intermediate risk (range: $0.0-8.3 \%$; $95 \%$ CI: 1.4-3.9\%). Among PWID, HCV incidence at 6 months was assessed at 66.7 per 100 person-years in one study. Geographic and temporal variations in HCV prevalence among PWID and prisoners appear to be present. While prevalence among PWID appeared to decline over few years from $36.0 \%$ to $27.6 \%$ in Kabul, from $12.5 \%$ to $9.5 \%$ in Jalalabad, and from $24.1 \%$ to $18.8 \%$ in Mazar-i-Sharif, it increased from $49.1 \%$ to $70 \%$ in Herat. Among prisoners, HCV prevalence appeared to increase from $1.7 \%$ to $4.6 \%$ in Kabul and declined from $4.1 \%$ to $1.4 \%$ in Herat. Risk factors among PWID included a range of injecting behaviours and socio-demographic characteristics.

Conclusions HCV prevalence among the general population in Afghanistan is comparable to developing and developed countries globally. There is an immediate need for increasing access to harm reduction programs among specific populations at risk, specifically PWID and prisoners.

Disclosure of interest statement This publication was made possible by NPRP grant number [NPRP 4-924-3-251] from the Qatar National Research Fund (a member of Qatar Foundation). Additional support was provided by the Biostatistics, Epidemiology, and Biomathematics Research Core at the Weill Cornell Medical College in Qatar. The statements made herein are solely the responsibility of the authors. The funders had no role in the design, conduct, or analysis of the study. The authors have no conflicts of interest to declare.

\section{P10.16 FACTORS ASSOCIATED WITH ACCEPTANCE OF GENITAL HERPES TESTING FOR BLACK PATIENTS PRESENTING FOR CARE AT AN STD CLINIC}

L Elopre*, N Van Wagoner, B Van Der Pol, M Whitfield, E Hook. Division of Infectious Diseases, Department of Medicine, University of Alabama at Birmingham, Birmingham AL

\subsection{6/sextrans-2015-052270.444}

Background $15.5 \%$ of the population in the United States is infected with herpes simplex virus-type 2 (HSV-2). HSV-2 rates are disproportionately high in black Americans, with seroprevalence approaching $50 \%$ in some communities. Knowledge of infection status is an important prevention tool. We evaluated barriers to acceptance of serological testing for HSV-2 in black patients presenting to an STD clinic.

Methods This ongoing study evaluates factors associated with HSV-2 serological test acceptance in patients living in the Southeastern US Participants did not report a history of genital herpes and data analyses were restricted to black patients $(93.5 \%$ of the study population). Sera were tested with HerpeSelect ${ }^{\circledR}$ assays. $\chi^{2}$ tests determined differences between groups based on testing. Univariate (UV) and multivariable (MV) analyses were performed to determine odds ratios (OR) for factors associated with test acceptance.

Results Of 112 participants, only 85 (75.9\%) accepted HSV-2 testing. Although only one participant felt their test results would be positive, seroprevalence for HSV-2 was 31.7\%. Test acceptance was more common in persons presenting for symptom evaluation $(53.1 \%)$ than as contacts $(17.2 \%)$ or for screening (29.7\%). In UV analyses, participants who reported depression (OR 2.8; 95\% CI 1.1, 8.3), previous HIV testing (OR 3.0; 95\% CI 1.1, 7.9) and STI history (OR 5.8; 95\% CI $1.5,24.4)$ were more likely to accept testing. Only previous HIV testing (AOR 7.4; 95\% CI 1.2, 66.4) remained significant in MV analysis.

Conclusions While many participants accepted testing, the rate was lower than in previous studies with little perception of risk. Culturally tailored interventions are needed to enhance assessment of HSV risk and test acceptance.

Disclosure of interest statement No disclosures.

\section{P10.17 INHIBITION OF HERPES SIMPLEX VIRUS 1 (HSV-1) REPLICATION BY INOSITOL-REQUIRING ENZYME 1 (IRE1) PATHWAY}

${ }^{1}$ AR Su*, 'XH Wang, ${ }^{1,2} \mathrm{ZW}$ Wu. 'Center for Public Health Research, Medical School, Nanjing University, Nanjing, PR China; ${ }^{2}$ State Key Lab of Analytical Chemistry for Life Science, Nanjing University, Nanjing, PR China

\subsection{6/sextrans-2015-052270.445}

Introduction Endoplasmic reticulum (ER) plays important roles in viral replication. Massive viral proteins entering the ER can trigger ER stress. The ER stress is marked by the activation of a series of signalling pathways, called the unfolded protein response (UPR), which consists of three distinct, yet related, signal pathways, PERK, IRE1 and ATF6. The PRKR-like ER kinase (PERK) signalling branch has been the focus of investigation on its roles during HSV gene expression. In contrast, there have been little studies on roles of the IRE1 branch of the UPR on HSV replication.

Methods Western blot, RT-PCR, RNA interference and in cell western method were used in the study.

Results We showed that HSV-1 replication was inhibited by the phosphorylation of the IRE1 (p-IRE1) pathway. We also detected the mRNA expression of spliced $\mathrm{X}$ box-binding protein 1 $\left(\mathrm{XBP} 1_{\mathrm{s}}\right)$, which was activated by $\mathrm{p}-\mathrm{IRE} 1$. IRE1 mRNA was upregulated by viral infection, but $\mathrm{XBP} 1_{\mathrm{s}}$ was not affected, which indicated that viral replication is not associated with spliced XBP1 signal pathway. It is known that cJUN NH2-terminal kinases JNK [JNKs; also known as stress-activated protein kinases (SAPKs)] is another downstream component of IRE1 and JNK is known to be activated by infected cell polypeptide 0 (ICP0), the immediate early protein of HSV-1. Our results revealed that the JNK activation was attenuated following the IRE1 inactivation. Inhibition of ire1 function by RNA interference (siRNA) could block the viral replication and JNK activation, suggesting that IRE1 pathway is required for facilitating viral gene expression and the inhibition of IRE1 activation blocks viral replication.

Conclusion The identification of roles of the IRE1 branch of ER stress in HSV-1 replication will provide new insight to the HSV1 pathogenesis and may lead to new therapeutic targets.

Disclosure of interest statement No conflict of interest in the development of this study.

\section{P10.18 ACYCLOVIR 1 GM TWICE A DAY FOR 3 DAYS FOR THE TREATMENT OF RECURRENT GENITAL HERPES}

K Verma*, M Sonune, S Gupta. Department of Dermatology and Venereology, All India Institute of Medical Sciences, New Delhi, India

\subsection{6/sextrans-2015-052270.446}

Background Recurrent genital herpes is conventionally treated with acyclovir $200 \mathrm{mg} 5$ times a day orally which is inconvenient to take. We studied the effectiveness and safety of acyclovir $1 \mathrm{gm}$ twice daily orally for 3 days in treatment of genital herpes. 